\title{
Flow over a square cylinder by BEM
}

\author{
L. Škerget \& J. Ravnik \\ University of Maribor, Faculty of Mechanical Engineering, \\ Maribor, Slovenia
}

\begin{abstract}
Flow of an incompressible viscous fluid is considered. The velocity-vorticity formulation of the Navier-Stokes equations is used. The kinematics equation is solved for boundary vorticity values using the Boit-Savart law. Solution of the kinetics equation for the domain values is obtained by employing a macro element approach. Using macro elements enables simulations on dense meshes, since it substantially reduces the algorithm's memory requirements.

The developed numerical algorithm has been used to simulate laminar flow over a square cylinder in channel. Low Reynolds number steady state flow simulation as well as transient simulation at higher Reynolds numbers has been investigated. The results have been analysed in terms of velocity, vorticity and pressure field distributions in the wake of the cylinder.
\end{abstract}

\section{Introduction}

Flows past bluff bodies are an interesting topic amongst engineers and researchers. A square cylinder is a basic example of such flows, its industrial applicability ranging from wind induced motion to turbulent sound generation. In general flows past bluff bodies exhibit complex phenomena such as separation, reattachment or vortex shedding.

The numerical benchmark for laminar incompressible flow over a square cylinder was made by Breuer et al. [1] using lattice Boltzmann and finite volume methods. Recently turbulent flow over a square cylinder was considered by Sohankar [2] using the Large eddy simulation approach. Flow as well as heat transfer was considered by De and Dalal [3] for a study of a natural convection around a headed square cylinder in an enclosure. Ozgoren [4] studied experimentally the flow structure in the downstream of square and circular cylinders. 
We have used the incompressible Navier-Stokes equations written in velocity vorticity formulation to solve flow past a square cylinder. In order to decrease the computer storage requirements of BEM, wavelet compression technique was used by Ravnik et al. [5]. In this paper we present results of an alternative approach - the equations were solved by the macro element Boundary Element Method (BEM). The derived equations, the numerical procedure and the results are shown below.

\section{The Navier-Stokes equations}

The analytical description of motion of a continuous incompressible and isothermal fluid medium is based on conservation of mass and momentum. The primitive field functions of interest are velocity vector field $\vec{v}(\vec{r}, t)$ and scalar pressure field $p(\vec{r}, t)$. The dynamics of a viscous fluid flow may be partitioned into its kinematic and kinetic aspect through the use of derived vector vorticity field $\vec{w}(\vec{r}, t)$, obtained as a curl of the compatible velocity field, as follows

$$
\omega_{i}=e_{i j k} \frac{\partial v_{k}}{\partial x_{j}}, \quad \frac{\partial \omega_{j}}{\partial x_{j}}=0,
$$

which is solenoidal vector by the definition, and $e_{i j k}$ is the permutation unit tensor. By applying a curl to the vorticity definition (1) and using the solenoidal constraint for the velocity vector $\vec{\nabla} \cdot \vec{v}=0$, the following vector elliptic Poisson's equation for the velocity vector is obtained

$$
\triangle \vec{v}+\vec{\nabla} \times \vec{\omega}=0
$$

The equation (2) represents the kinematics of an incompressible fluid motion, expressing the compatibility and restriction conditions between velocity and vorticity field functions.

The kinetic aspect is governed by the parabolic diffusion convection vorticity equation, obtained by applying the curl differential operator to the momentum equation. For the two-dimensional plane flow, the vorticity vector has just one component, which is perpendicular to the plane of the fluid motion. Thus we obtain a scalar transport equation for vorticity

$$
\frac{D \omega}{D t}=\nu \triangle \omega
$$

being $\nu$ constant kinematic viscosity and $D / D t$ Stokes substantial derivative.

The vorticity transport equation as itself is highly non-linear partial differential equation due to the inherent non-linearity caused by the compatibility and restriction conditions among velocity and vorticity fields, and due to the product of velocity and vorticity field functions in the convective term. 
To derive the pressure equation, depending on known velocity and vorticity field functions, the divergence of momentum equation should be considered, resulting in the elliptic Poisson's pressure equation

$$
\triangle p-\vec{\nabla} \cdot \overrightarrow{f_{p}}=0
$$

where the pressure force term $\vec{f}_{p}$ is for the planar flow cases

$$
\frac{\partial p}{\partial x_{i}}=f_{p i}=-\eta e_{i j} \frac{\partial \omega}{\partial x_{j}}-\rho a_{i}+\rho g_{i}
$$

in which the dynamic viscosity is $\eta=\rho \nu$, and $\vec{a}=D \vec{v} / D t$ and $\vec{g}$ are the inertia and gravitational acceleration vectors, respectively. The Neumann boundary conditions for the pressure equation may be determined for the whole solution boundary $\Gamma$ and the following relation is valid

$$
\frac{\partial p}{\partial n}=\overrightarrow{f_{p}} \cdot \vec{n}
$$

\section{Boundary-domain integral representations}

To apply the boundary element method, we must rewrite the governing differential equations in integral form. The singular boundary-domain integral representation for the velocity vector can be formulated by using the Green theorems for scalar functions or weighting residuals technique rendering the following vector integral formulation, e.g. the plane two-dimensional kinematics is given by two scalar equations as follows [4] (Škerget et al. [6])

$$
c(\xi) v_{i}(\xi)+\int_{\Gamma} v_{i} \frac{\partial u^{\star}}{\partial n} d \Gamma=e_{i j} \int_{\Gamma} v_{j} \frac{\partial u^{\star}}{\partial t} d \Gamma-e_{i j} \int_{\Omega} \omega \frac{\partial u^{\star}}{\partial x_{j}} d \Omega,
$$

where $u^{\star}$ is the elliptic Laplace fundamental solution, $\xi$ the source point and $\Gamma$ the boundary of the solution domain $\Omega$.

Considering the vorticity kinetics in an integral representation one has to take into account parabolic diffusion convection character of the vorticity transport equation [3]. With the use of the linear parabolic diffusion-convective differential operator, the following integral formulation can be written (Škerget et al. [7])

$$
\begin{aligned}
c(\xi) \omega(\xi) & +\int_{\Gamma} \omega \frac{\partial U^{\star}}{\partial n} d \Gamma=\int_{\Gamma} \frac{\partial \omega}{\partial n} U^{\star} d \Gamma-\frac{1}{\nu} \int_{\Gamma} v_{n} \omega U^{\star} d \Gamma \\
& +\frac{1}{\nu} \int_{\Omega} \widetilde{v}_{j} \omega \frac{\partial U^{\star}}{\partial x_{j}} d \Omega+\beta \int_{\Omega} \omega_{F-1} U^{\star} d \Omega,
\end{aligned}
$$

where the velocity field is decomposed into an average constant vector $\bar{v}_{j}$ and a perturbated one $\widetilde{v}_{j}$, such that $v_{j}=\bar{v}_{j}+\widetilde{v}_{j}$, the quantity $U^{\star}=\nu u^{\star}$ with $u^{\star}$ is now the fundamental solution of diffusion-convective equation with first order reaction, and $\beta=1 / \nu \triangle t$. 
The pressure equation (4) is an elliptic Poisson equation, and therefore employing again the linear elliptic Laplace differential operator [5] the following form of the pressure integral equation is obtained (Škerget and Samec [8])

$$
c(\xi) p(\xi)+\int_{\Gamma} p \frac{\partial u^{\star}}{\partial n} d \Gamma=\int_{\Omega} f_{p i} \frac{\partial u^{\star}}{\partial x_{i}} d \Omega,
$$

where the vector $\overrightarrow{f_{p}}$ is given by equation (5).

\section{Numerical example: flow over a square cylinder}

The developed numerical algorithm was used to simulate incompressible laminar 2D flow over a square cylinder. The cylinder was positioned in a centre of a channel. A parabolic velocity profile was prescribed at the inflow of the channel. At the channel walls and at the cylinder a no slip boundary condition was applied. At the outflow boundary normal derivatives of all field functions were set to zero. Boundary and initial conditions are shown schematically in Figure 1. The flow configuration was the same as in Camarri and Giannetti [9] with the Reynolds number valued defined by $R e=D v_{c} / \nu$. The blockage ratio was $\beta=D / H=$ $1 / 8$. The length of the flow domain before the cylinder was $L_{i} / D=12$ and behind the cylinder $L_{o} / D=35$.

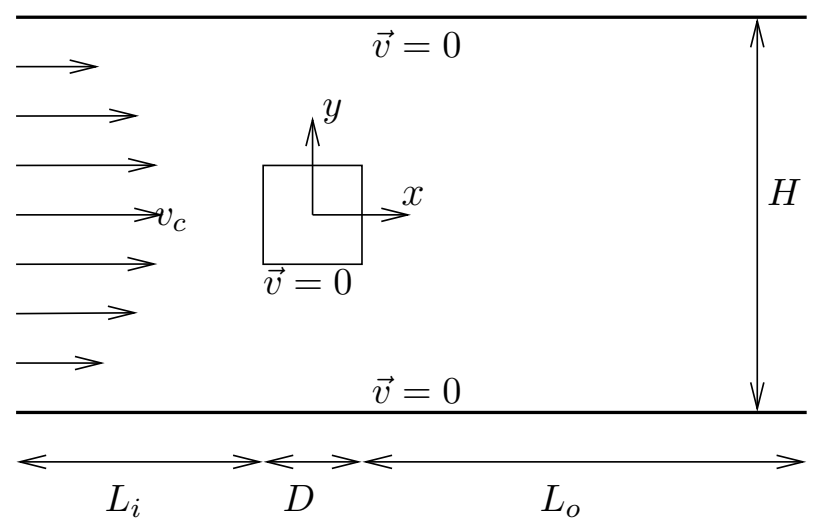

Figure 1: Flow configuration, boundary conditions and computational domain, not in scale.

The simulation was performed on a computational mesh consisting of 4700 Lagrangian 9 node domain cells. The mesh is illustrated in Figure 2.

The flow was simulated for Reynolds number values from $R e=9$ to $R e=90$. Flow at $R e=9, R e=18$ and $R e=36$ is steady. Steady state simulation was used to obtained converged results for $R e=9$ and $R e=18$. At $R e=36$ transient 


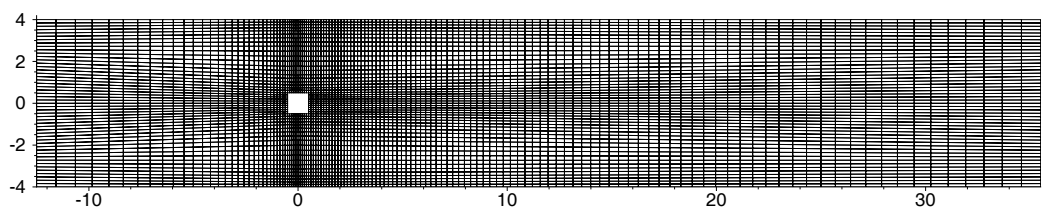

Figure 2: Computational mesh for the simulation of flow over a square cylinder. 4700 Lagrangian domain cells were used.

simulation was performed, having the steady state $R e=18$ results for initial conditions. A time step of $\Delta t=0.1$ was used. The simulation ran 140 time steps to achieve steady state. The contours of vertical and horizontal velocity, vorticity and pressure are shown in Figure 3 for $R e=9$, in Figure 4 for $R e=18$ and in in Figure 5 for $R e=36$. One may observe that the recirculation region behind the cylinder grows with the increasing Reynolds number. The flow speeds up between the cylinder and the walls of the channel, while it slows in front of and behind the cylinder. Looking at vorticity contours we observe that the largest vorticity can be found at the lower left and upper left corner of the cylinder. Vorticity is generated at solid walls and is transported by diffusion and advection into the flow. Looking at the pressure field, we notice the high pressure zone in front of the cylinder and a low pressure zone at the top, bottom and behind the cylinder.

At Reynolds number value $R e=90$ the flow becomes unsteady. A time step of $\Delta t=0.1$ was used. The von Kármán vortex street is formed behind the cylinder, i.e. clockwise and counter-clockwise vortices are shed from the upper and lower sides of the cylinder. Figure 6 shows the instantaneous contours of vertical and horizontal velocity, vorticity and pressure at $t=30$.

Breuer et al. [1] and Camarri and Giannetti [9] reported the Strouhal number $\left(S t=f D / v_{c}, \mathrm{f}\right.$ being the vortex-shedding frequency) to be $S t=0.135$ at $R e=90$. By observing vorticity behind the cylinder at $x=0.968, y=0$ (Figure 7) we were able to measure the vortex shedding frequency $f=0.2$. Using the measure shedding frequency we calculated the Strouhal number $S t=0.133$, which is in excellent agreement with the reference results.

\section{Conclusions}

A macro element boundary element method based method was developed for the simulation of two-dimensional laminar incompressible viscous fluid flows. The method was tested on a flow over a square cylinder confined in a channel.

Incompressible flow past a square cylinder is steady for Reynolds number values $R e=9, R e=18, R e=36$ with the length of the recirculation region increasing with the increasing Reynolds number. At Reynolds number value $R e=90$ we simulated unsteady behaviour. Vortices are shed from the upper and lower walls of the cylinder. The Strouhal number exhibited by the flow using our simulation was $S t=0.133$, which in excellent agreement with the benchmark results. 
166 Boundary Elements and Other Mesh Reduction Methods XXIX

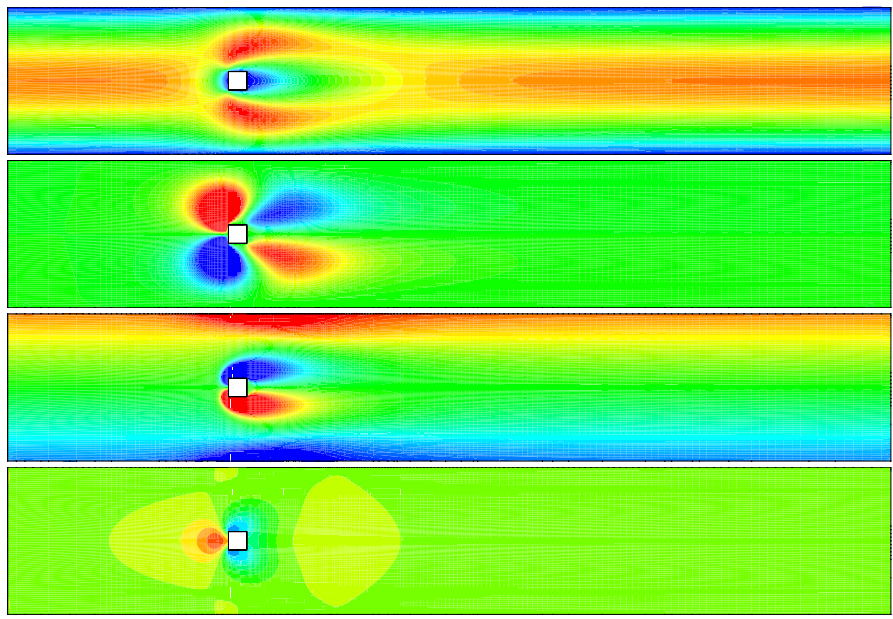

Figure 3: Steady state flow over a square cylinder at $R e=9$. From top to bottom: horizontal velocity, vertical velocity, vorticity and pressure contours.

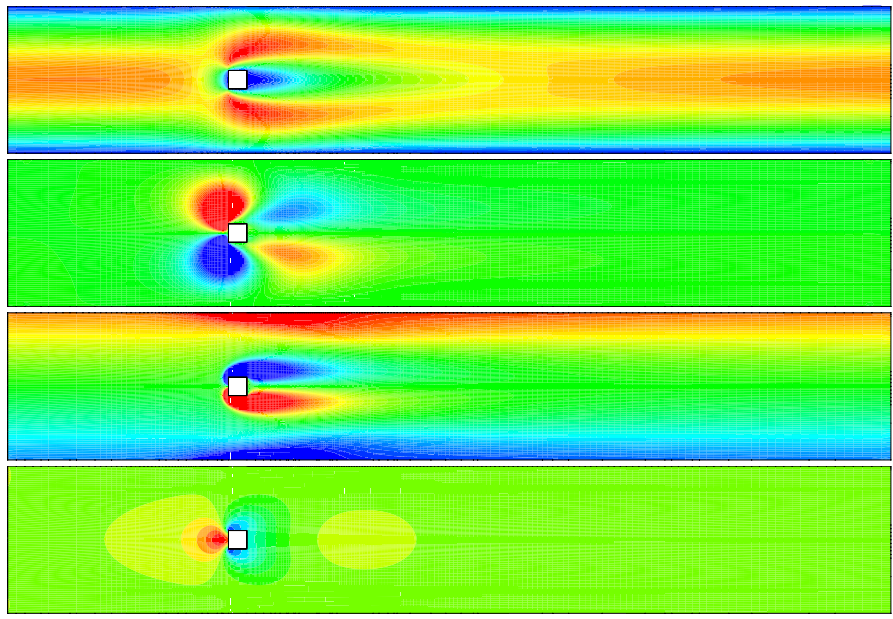

Figure 4: Steady state flow over a square cylinder at $R e=18$. From top to bottom: horizontal velocity, vertical velocity, vorticity and pressure contours. 


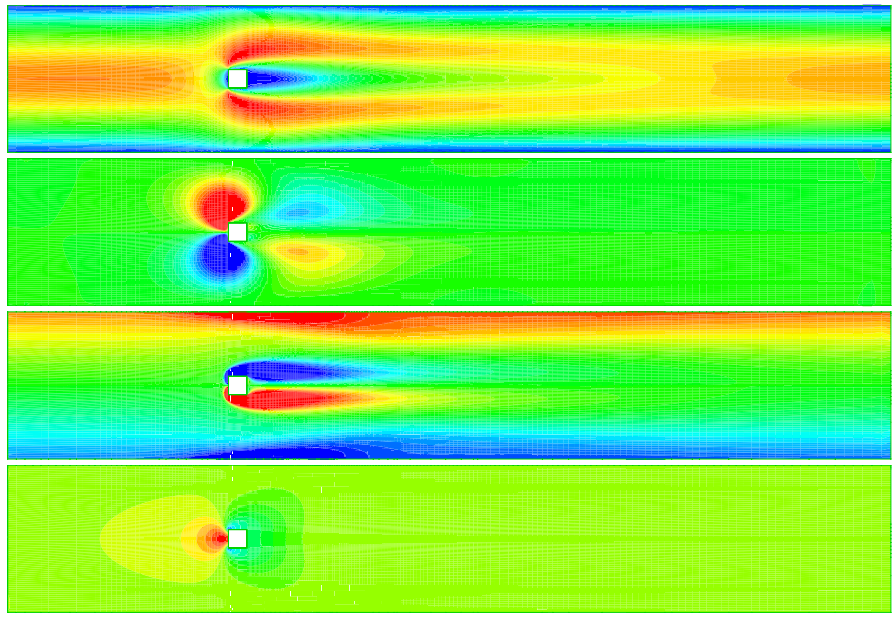

Figure 5: Steady state flow over a square cylinder at $R e=36$. From top to bottom: horizontal velocity, vertical velocity, vorticity and pressure contours.

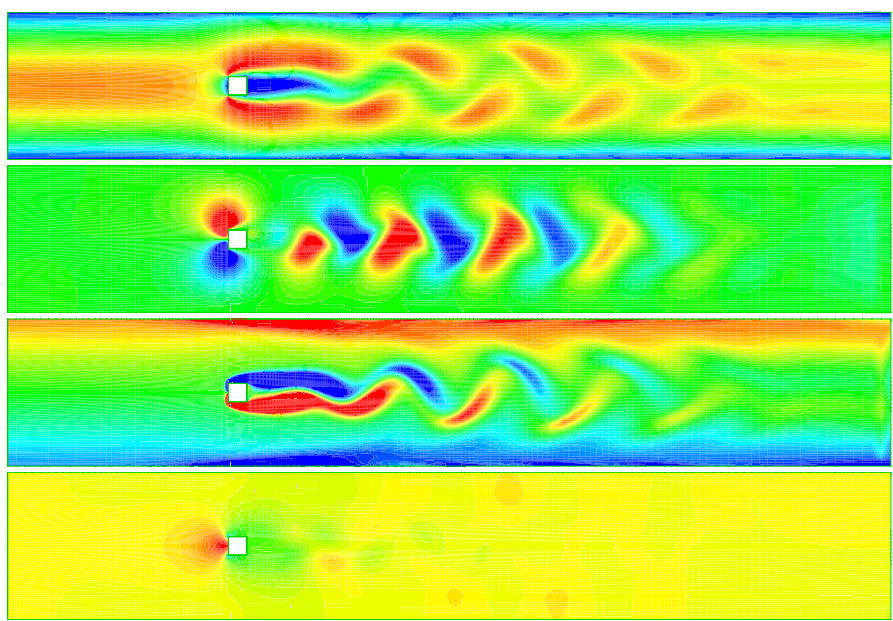

Figure 6: Unsteady flow over a square cylinder at $R e=90, t=30$. From top to bottom: horizontal velocity, vertical velocity, vorticity and pressure contours. 


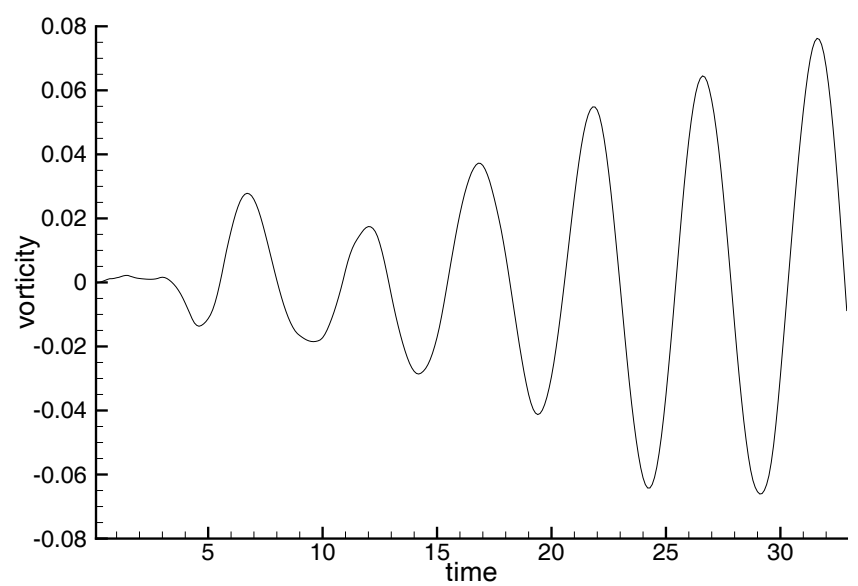

Figure 7: Flow over a square cylinder at $R e=90$. Vorticity at $x=0.968, y=0$ was used to measure the Strouhal number.

\section{References}

[1] Breuer, M., Bernsdorf, J., Zeiser, T. \& Durst, F., Accurate computations of the laminar flow past a square cylinder based on two different methods: latticeBoltzmann and finite-volume. Int J Heat and Fluid Flow, 21, pp. 186-196, 2000.

[2] Sohankar, A., Flow over a bluff body from moderate to high reynolds numbers using large eddy simulation. Computers \& Fluids, 35, pp. 1154-1168, 2006.

[3] De, A.K. \& Dalal, A., A numerical study of natural convection around a square, horizontal, heated cylinder placed in an enclosure. Int J Heat Mass Transf, 49, pp. 4608-4623, 2006.

[4] Ozgoren, M., Flow structure in the downstream of square and circular cylinders. Flow Measurement and Instrumentation, 17, pp. 225-235, 2006.

[5] Ravnik, J., Škerget, L. \& Hriberšek, M., 2D velocity vorticity based LES for the solution of natural convection in a differentially heated enclosure by wavelet transform based BEM and FEM. Eng Anal Bound Elem, 30, pp. 671686, 2006.

[6] Škerget, L., Hriberšek, M. \& Žunič, Z., Natural convection flows in complex cavities by BEM. Int J Num Meth Heat \& Fluid Fl, 13, pp. 720-735, 2003.

[7] Škerget, L., Hriberšek, M. \& Kuhn, G., Computational fluid dynamics by boundary domain integral method. Int J Num Meth Eng, 46, pp. 1291-1311, 1999.

[8] Škerget, L. \& Samec, N., BEM for the two-dimensional plane compressible fluid dynamics. Eng Anal Bound Elem, 29, pp. 41-57, 2005.

[9] Camarri, S. \& Giannetti, F., Analysis of the inversion of the von Kármán street in the wake of a confined square cylinder. EUROMECH Fluid Dynamics Conference, pp. 19-25, 2006. 